\title{
Analysis and Water Quality Control of Alternative Sources in Bangolan, Northwest Cameroon
}

\author{
Nchofua Festus Biosengazeh $\mathbb{D},{ }^{1}$ Njoyim Estella Buleng Tamungang $\mathbb{D},{ }^{1,2}$ \\ Mofor Nelson Alakeh $\mathbb{D}^{1,3}$ and Mvondo-ze Antoine david ${ }^{4}$ \\ ${ }^{1}$ Research Unit of Noxious Chemistry and Environmental Engineering (RUNOCHEE), Department of Chemistry, \\ Faculty of Science, University of Dschang, Dschang, Cameroon \\ ${ }^{2}$ Department of Chemistry, Higher Teacher Training College, The University of Bamenda, Bambili, Cameroon \\ ${ }^{3}$ Department of Mining and Mineral Engineering, National Higher Polytechnic Institute, The University of Bamenda, \\ Bambili, Cameroon \\ ${ }^{4}$ Research Unit of Soil Analysis and Environmental Chemistry (RUSAEC), Department of Soil Science, \\ Faculty of Agronomy and Agricultural Sciences (FAAS), University of Dschang, Dschang, Cameroon
}

Correspondence should be addressed to Nchofua Festus Biosengazeh; fnchofua@yahoo.com

Received 16 December 2019; Revised 15 April 2020; Accepted 2 May 2020; Published 25 May 2020

Academic Editor: Xi Liu

Copyright (c) 2020 Nchofua Festus Biosengazeh et al. This is an open access article distributed under the Creative Commons Attribution License, which permits unrestricted use, distribution, and reproduction in any medium, provided the original work is properly cited.

\begin{abstract}
This research focused on the quality of domestic water in Bangolan, Northwest Cameroon, in order to ascertain its potability based on World Health Organisation (WHO) guidelines. Inhabitants of this locality, as well as others in rural areas, consume water from these sources without any prior treatment which can lead to contamination and infections. Sampling was conducted, and physicochemical and bacteriological properties of ten water sources were examined in November 2017 and January, April, and July 2018 using standard methods. The results showed that the sampled water sources were moderately acidic to weakly basic falling within the WHO specification. The values of electrical conductivity and total dissolved solids were low, suggesting low mineralised waters which could lead to a shortage of essential mineral elements in humans. Water turbidity was high in July, attributable to the rains. Major ions analysed fell within the WHO guideline values except iron and aluminium, whose values fell above guideline values in both seasons due to the nature of the soils. Faecal coliforms were found in all the sources, and specific bacteria identified were Escherichia coli, Enterobacteria, Streptococcus, Salmonella, and Shigella spp., suggesting recent contamination by faecal matter due to poor hygienic conditions, and hence, treatment was recommended. Statistical analyses revealed significant influence of heavy rains on most water parameters $(p<0.05)$. Health data in the locality revealed 1389 cases of waterborne diseases, namely, typhoid, diarrhoea, and dysentery between 2016 and 2017 necessitating control.
\end{abstract}

\section{Introduction}

About $80 \%$ of the Earth's surface is covered by water, but freshwater supply has increasingly become a major problem [1]. Water-related diseases caused by insufficient safe water supplies, coupled with poor sanitation and hygiene, are responsible for 3.4 million deaths every year, mostly among children $[2,3]$. For this reason, water quality assessment and continuous monitoring are of utmost importance [4]. National drinking water standards often stipulate the maximum permissible concentration of contaminants in drinking water, and at a global scale, the World Health Organization (WHO) guidelines provide recommendations for managing the risk from hazards that may compromise the safety of drinking water $[5,6]$. In spite of the success of the Millennium Development Goals (MDGs) water target, 663 million people, with 319 million in sub-Saharan Africa of which $80 \%$ live in rural areas, still lack improved drinking water sources and 2.5 billion people are without access to an improved sanitation facility [7]. 
The importance of water, sanitation, and hygiene for health and development has continued to receive attention worldwide. This comprises the recent adoption of the Sustainable Development Goals (SDGs) by the United Nations (UNs) in 2015. Through goal 6 of the SDGs which is to ensure availability and sustainable management of water and sanitation for all, the UNs reaffirm its commitment in ensuring safe drinking water and sanitation to the global population by $2030[8,9]$. With a growing demand for water and other related issues, it is likely that the SDGs target may not be met in sub-Saharan Africa countries, with Cameroon included.

Cameroon is the second country in Africa (after the Democratic Republic of Congo) in terms of quantity of available water resources estimated to be 322 billion cubic meters. This gives an annual available water per inhabitant of $21,000 \mathrm{~m}^{3}$ [10]. Today, the major difficulty with which Cameroonians are confronted is not so much access to water but more precisely the access to suitable water for domestic uses as water-related diseases represent about two-thirds of all the diseases in this country and are responsible for approximately $50 \%$ of death cases recorded annually $[11,12]$. Improved drinking water sources and sanitation facilities in semiurban and rural communities of the country is only $49 \%$ [7]. In rural areas where conventional water network is absent, the populations rely on community taps, surface, and groundwater sources for domestic uses. The quality of these sources is largely determined by both natural processes (dissolution and precipitation of minerals, groundwater velocity, quality of recharge waters, and interaction with other types of water aquifers) and anthropogenic activities [1].

In Bangolan, pipe-borne water is provided to the population by the council and managed by a community water committee. In addition, the population also uses water from streams, wells, springs, and manual pump boreholes with little available information on their physicochemical and bacteriological properties. The available taps are found in very few quarters, and water from it is sometimes coloured. Most of the wells are shallow, not properly protected, suggesting possible infiltrations of dirty water, urine, or excrement into the water body. The boreholes are currently out of use due to poor maintenance. As for the springs, they are not properly taken care of as they are exposed to dust, close to farms, often shared with animals, and are exposed to floods in the rainy season, suggesting their contamination by faeces and fertilizers. Suitability of these water sources for domestic use is thus a serious concern, and there arises the need for proper quality assessment and monitoring. Data obtained from health centres in the community revealed 1389 cases of water-borne infections, namely, typhoid, diarrhoea, and dysentery between 2016 and 2017 giving 9\% infected cases within the entire population during this period [13].

The main objective of this study was thus to examine the quality of water from various sources in Bangolan in order to ascertain its suitability for domestic uses based on WHO standards and to evaluate seasonal impacts on water properties. To achieve this objective, water samples were analysed for physical, chemical, heavy metals content, and bacteriological properties, and recommendations were made based on the findings obtained.

\section{Materials and Methods}

2.1. Description of the Sampling Site. Bangolan is found in Babessi subdivision under Ngo-Ketunjia Division in the Northwest Region of Cameroon (Figure 1) situated at the border of lake Bamendjing and the west region in the south and at about $61 \mathrm{~km}$ from Bamenda, Bangolan, lies between latitude $5.978563^{\prime}$ north and longitude $10.623045^{\prime}$ east with an estimated population of 15000 inhabitants. The community is supplied by two spring catchments, and the pipeborne water project is almost nonfunctional, and more so, it covers just some few households in the village [14]. The main activities of the inhabitants are agriculture, breeding, and small-scale trading. The climate is characterized by a short dry season from November to February and a long raining season from March to October. The annual maximum temperature varies between $27.2^{\circ} \mathrm{C}$ and $33.6^{\circ} \mathrm{C}$, whereas the annual minimal temperature varies between $7.8^{\circ} \mathrm{C}$ and $15.9^{\circ} \mathrm{C}$. Pluviometry varies between $1270 \mathrm{~mm}$ and $1778 \mathrm{~mm}$ of water per year. Its hydrology is characterized by the existence of small rivers, streams, springs, and swamps. The ground is mainly basaltic, trachytic, and/or granitic favourable for agriculture and pasture. Vegetation is mainly savannah type with short stunted trees [15].

2.2. Sampling and Preservation. Samples were collected from ten sources in November 2017 and January, April, and July 2018. At each sampling point, three samples were collected in clean and labelled polyethylene containers of $500 \mathrm{~mL}$ capacity. Samples for heavy metals analysis were preserved with concentrated nitric acid. The containers and caps were thoroughly rinsed with water to be sampled before collection. The collections were done very early in the morning before sunrise, and the samples were packaged in a cooler containing ice in order to maintain the temperature at $4^{\circ} \mathrm{C}$ [16]. Finally, the samples were transported to the Research Unit of Animal Physiology and Microbiology and the Research Unit of Soil Analysis and Environmental Chemistry of the University of Dschang for preservation and analyses. A global positioning system (GPS) (Garmin Etrex Vista) was used to locate the study site. The source type, sample code, and their geographical coordinates are presented in Table 1.

\subsection{Analyses}

2.3.1. Physicochemical Analyses. Temperature (T), $\mathrm{pH}$, electrical conductivity (EC), and total dissolved solids (TDS) were measured in situ with the help of a calibrated multimeter, HANNAH198128. Turbidity was measured using a turbidimeter (Model DRT, 100B, MF scientific, Inc). Chloride content was determined by the argentometric method. Nitrate and ammonium were determined by Kjeldahl's distillation method. Phosphates were determined by UV-visible spectrophotometric analysis. Bicarbonates 


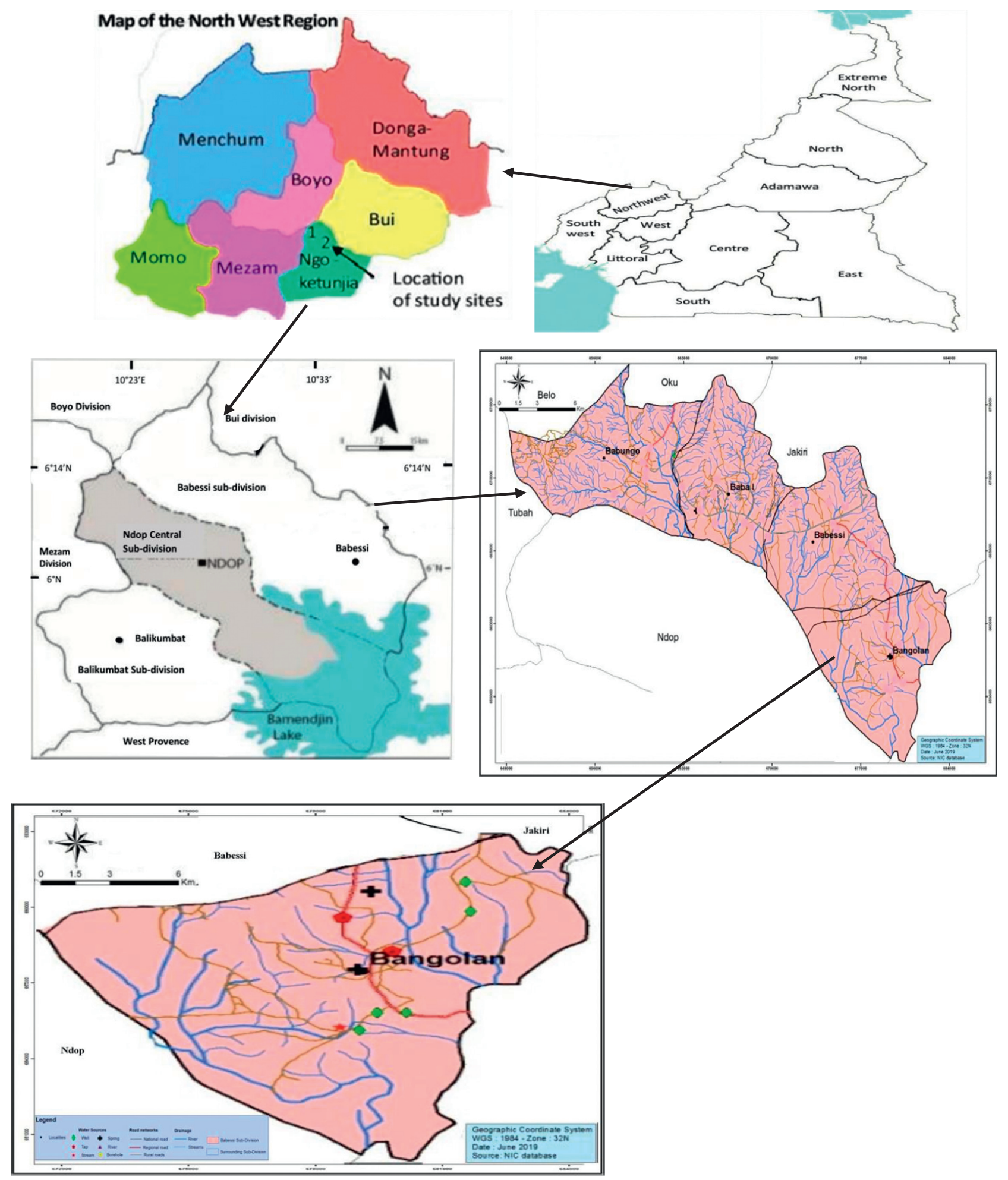

Figure 1: Map of the study area: (a) map of Cameroon, indicating the map of northwest region; (b) map of northwest region indicating the map of Ngo- Ketunjia; (c) map of Ngo-Ketunjia indicating the map of Babessi subdivision; (d) map of Babessi subdivision indicating the map of Bangolan; (e) map of Bangolan showing the sampling points.

were determined by acid-base titration and sulphates by gravimetric analysis. $\mathrm{Ca}$ and $\mathrm{Mg}$ ions were determined by complexometric titration, while $\mathrm{Na}$ and $\mathrm{K}$ were determined by flame photometry. $\mathrm{Zn}, \mathrm{Al}, \mathrm{Fe}, \mathrm{Pb}$, and $\mathrm{Cu}$ were determined by colorimetry following methods described by Rodier et al. [16] and WHO [6]. 
TABLE 1: Quarters, source type, sample codes, and geographical coordinates of sampling points.

\begin{tabular}{|c|c|c|c|c|}
\hline Quarter & Source type & Code & Coordinates & Elevation $(\mathrm{m})$ \\
\hline Nstotchimbere & Stream & STnts & $\mathrm{N}^{\circ} 5^{\circ} 55^{\prime} 43,5^{\prime \prime} \mathrm{E} 010^{\circ} 36^{\prime} 59,1^{\prime \prime}$ & 1167 \\
\hline Nchotchimbere & Well & Wnts & N05 $55^{\prime} 39,4^{\prime \prime}$ E $010^{\circ} 37^{\prime} 12,3^{\prime \prime}$ & 1119 \\
\hline Nkwaliang & Well & Wnkw & $\mathrm{N} 05^{\circ} 56^{\prime} 01,0^{\prime \prime} \mathrm{E} 010^{\circ} 37^{\prime} 44,4^{\prime \prime}$ & 1076 \\
\hline Mbuntoh & Well & $\mathrm{Wmbu}$ & N05 $56^{\prime} 17,0^{\prime \prime}$ E $010^{\circ} 37^{\prime} 24,7^{\prime \prime}$ & 1094 \\
\hline Makulung & Spring & SPmak & $\mathrm{N} 05^{\circ} 58^{\prime} 30,5^{\prime \prime} \mathrm{E} 010^{\circ} 37^{\prime} 21^{\prime \prime}$ & 1167 \\
\hline Ngoliang & Tap & Tngo & $\mathrm{N} 05^{\circ} 57^{\prime} 16,7^{\prime \prime} \mathrm{E} 010^{\circ} 37^{\prime} 35,3^{\prime \prime}$ & 1160 \\
\hline Njang & Well & Wnja & $\mathrm{N} 05^{\circ} 58^{\prime} 05,1^{\prime \prime} \mathrm{E} 010^{\circ} 38^{\prime} 28,5^{\prime \prime}$ & 1166 \\
\hline Njang & Well & Wnja1 & N05 $58^{\prime} 41,7^{\prime \prime}$ E $010^{\circ} 38^{\prime} 25,3^{\prime \prime}$ & 1087 \\
\hline Nkendeh & Tap & Tnk & N05 $57^{\prime} 58,7^{\prime \prime}$ E $010^{\circ} 37^{\prime} 01,5^{\prime \prime}$ & 1108 \\
\hline Nkendipeh & Spring & SPnke & $\mathrm{N}^{\circ} 5^{\circ} 56^{\prime} 54,6^{\prime \prime} \mathrm{E} 010^{\circ} 37^{\prime} 11,2^{\prime \prime}$ & 1160 \\
\hline
\end{tabular}

2.3.2. Bacteriological Analyses. Two methods were used: the multiple tube fermentation or the most probable number (MPN) technique for the presumptive determination of faecal coliforms and the standard count plate technique for determination of specific bacteria, as described by WHO [17] and Nanfack et al. [18].

2.3.3. Statistical Analyses. The data obtained from this study were subjected to general descriptive statistics and presented as mean \pm standard deviation (minimum-maximum). One-way analysis of variance (ANOVA) was used to explore seasonal impacts on water quality at 95\% confidence interval, post hoc comparisons were also performed using the Tukey HSD test, and Pearson correlation was used to verify relationships between water parameters. Analyses were performed with the help of Statistical Package for Social Sciences (SPSS) version 20.0 and Microsoft excel.

\section{Results and Discussion}

3.1. Results. The results of physical and chemical (cations, anions, and heavy metals) parameters of analysed water samples are presented in Tables $2-5$, respectively.

Results of physical parameters are presented in Table 2. The mean values of physical characteristics of water samples from the water sources over the study period showed that temperature ranged from $18.85 \pm 1.55^{\circ} \mathrm{C}$ (STnts) to $21.90 \pm 1.37^{\circ} \mathrm{C}$ (Wnja1). A maximum temperature of $23.80^{\circ} \mathrm{C}$ was recorded in Wnja in July 2018, while the least temperature $\left(17.10^{\circ} \mathrm{C}\right)$ was recorded in STnts in November 2017. $\mathrm{pH}$ ranged from $6.30 \pm 0.37$ (Wnts) to $7.67 \pm 0.32$ (STnts). Wnts had the least $\mathrm{pH}$ (5.80) in April 2018, while Wnkw and Wmbu had the highest pH values (8.00) in July 2018. Electrical conductivity and TDS values of all the water sources were very low and ranged from $10.78 \pm 3.86 \mu \mathrm{S} / \mathrm{cm}$ (Wnja) to $33.58 \pm 9.53 \mu \mathrm{S} / \mathrm{cm}(\mathrm{Wmbu})$ and $7.43 \pm 2.74 \mathrm{mg} / \mathrm{L}$ (Wnja) to $22.90 \pm 5.61 \mathrm{mg} / \mathrm{L}$ (Wmbu), respectively. Turbidity ranged from $0.88 \pm 0.78 \mathrm{NTU}$ (Tnk) to $6.88 \pm 2.96 \mathrm{NTU}$ (SPnke).

Results of chemical parameters analysed in the water samples are presented in Tables 3 and 4. The ions analysed included major water minerals, ammonium, and phosphates. The mean values of chemical characteristics of the water samples over the study period showed that calcium and magnesium concentrations ranged from
$21.25 \pm 7.72 \mathrm{mg} / \mathrm{L}$ (SPmak) to $43.00 \pm 13.32 \mathrm{mg} / \mathrm{L}$ (Tnk) and $3.03 \pm 2.83 \mathrm{mg} / \mathrm{L}$ (Wmbu) to $10.05 \pm 5.51 \mathrm{mg} / \mathrm{L}$ (SPnke) and were below the WHO guideline values of $75 \mathrm{mg} / \mathrm{L}$ and $30 \mathrm{mg} / \mathrm{L}$, respectively. $\mathrm{Na}^{+}, \mathrm{K}^{+}, \mathrm{Cl}^{-}, \mathrm{HCO}_{3}^{-}, \mathrm{SO}_{4}^{2-}$, $\mathrm{NO}_{3}^{-} \cdot \mathrm{NH}_{4}^{+}$, and $\mathrm{PO}_{4}^{3-}$ were all found in low concentrations and fell within the following ranges, respectively: $0.36 \pm 0.37 \mathrm{mg} / \mathrm{L} \quad$ (Wnja) to $1.52 \pm 1.23 \mathrm{mg} / \mathrm{L}$ (STnts), $1.21 \pm 0.66 \mathrm{mg} / \mathrm{L} \quad(\mathrm{Wmbu})$ to $2.68 \pm 1.39 \mathrm{mg} / \mathrm{L}$ (SPnke), $0.04 \pm 0.04 \mathrm{mg} / \mathrm{L}$ (SPmak and Wnja) to $0.52 \pm 0.58 \mathrm{mg} / \mathrm{L}$ (Tnk), $\quad 10.74 \pm 6.59 \mathrm{mg} / \mathrm{L}$ (SPmak) to $43.03 \pm 15.73 \mathrm{mg} / \mathrm{L}$ (Tnk), $\quad 0.35 \pm 0.35 \mathrm{mg} / \mathrm{L} \quad(W n k w)$ to $1.43 \pm 1.85 \mathrm{mg} / \mathrm{L}$ (SPnke), $\quad 0.62 \pm 0.84 \mathrm{mg} / \mathrm{L} \quad(W n k w)$ to $2.24 \pm 2.32 \mathrm{mg} / \mathrm{L}$ (SPmak), $0.43 \pm 0.62 \mathrm{mg} / \mathrm{L} \mathrm{(Wmbu)} \mathrm{to} 1.77 \pm 2.09 \mathrm{mg} / \mathrm{L}$ (SPnke), and $0.02 \pm 0.02 \mathrm{mg} / \mathrm{L}(\mathrm{Wnkw})$ to $1.72 \pm 1.83 \mathrm{mg} / \mathrm{L}$ (SPnke).

Heavy metals analysed included iron, lead, zinc, copper, and aluminium. The mean values of heavy metals concentrations (Table 5) showed that iron ranged from $0.01 \pm 0.01 \mathrm{mg} / \mathrm{L}$ (Wnts) to $1.76 \pm 1.03 \mathrm{mg} / \mathrm{L}$ (SPnke), zinc ranged from $0.55 \pm 0.34 \mathrm{mg} / \mathrm{L}$ (Wnja) to $2.26 \pm 1.17 \mathrm{mg} / \mathrm{L}$ (Tnk), aluminium ranged from $0.14 \pm 0.14 \mathrm{mg} / \mathrm{L}$ (Wnja) to $0.52 \pm 0.20 \mathrm{mg} / \mathrm{L}$ (Tnk), copper ranged from $0.13 \pm 0.14 \mathrm{mg} / \mathrm{L}$ (Tngo) to $1.37 \pm 0.59 \mathrm{mg} / \mathrm{l}$ (Wnkw), lead was below detectable limits in all the samples except SPnke with a mean value of $0.003 \pm 0.005 \mathrm{mg} / \mathrm{L}$.

The results of bacteriological analysis are presented in Figures 2-7. Faecal coliforms were found in all the water samples with most probable number of coliforms ranging from 11 to $30 / 100 \mathrm{~mL}, 8$ to $50 / 100 \mathrm{~mL}, 12$ to $90 / 100 \mathrm{~mL}$, and 30 to $160 / 100 \mathrm{~mL}$ in November 2017, and January, April, and July 2018, respectively. Based on WHO classification, all the water sources were unacceptable for human consumption in November as they showed a high risk and thus fell in category C. In January 2018, two of the sources precisely Wnts and $\mathrm{Wmbu}$ were found to be grossly polluted falling in category $\mathrm{D}$; the two springs (SPmark and SPnke) were acceptable for consumption classified as category B, while the remaining sources were in category C. In April 2018, three of the sources (STnts, Wmbu, and SPnke) fell in category $\mathrm{D}$, while the others were in category C. Meanwhile, in July 2018, all the sources were under category D except Tngo, Wnja1, and Tank which were classified in category C. Detailed investigations of the water samples also revealed the presence of specific bacteria among which were Enterobacteria spp., Escherichia coli, Streptococcus spp., Salmonella spp., 
TABLE 2: Physical parameters of samples from ten water sources in Bangolan within the study period.

\begin{tabular}{|c|c|c|c|c|c|}
\hline \multirow{2}{*}{$\begin{array}{l}\text { Sample } \\
\text { codes }\end{array}$} & \multicolumn{5}{|c|}{ Parameters } \\
\hline & $\mathrm{T}\left({ }^{\circ} \mathrm{C}\right)$ & $\mathrm{pH}$ & $\mathrm{EC}(\mu \mathrm{S} / \mathrm{cm})$ & TDS (mg/L) & Tur (NTU) \\
\hline STnts & $\begin{array}{c}18.85 \pm 1.55 \\
(17.10-20.30)\end{array}$ & $\begin{array}{l}7.67 \pm 0.32 \\
(6.80-7.90)\end{array}$ & $23.57 \pm 6.49(16.30-32.10)$ & $\begin{array}{c}16.23 \pm 3.84 \\
(11.60-21.00)\end{array}$ & $\begin{array}{c}3.52 \pm 4.47 \\
(0.90-10.20)\end{array}$ \\
\hline Wnts & $\begin{array}{c}20.40 \pm 1.67 \\
(19.00-22.50)\end{array}$ & $\begin{array}{l}6.30 \pm 0.37 \\
(5.80-6.70)\end{array}$ & $\begin{array}{c}19.70 \pm 4.73 \\
(14.20-24.40)\end{array}$ & $\begin{array}{c}13.90 \pm 3.24 \\
(10.20-17.30)\end{array}$ & $1.40 \pm 0.59(0.70-2.10)$ \\
\hline Wnkw & $\begin{array}{c}20.55 \pm 1.96 \\
(18.10-22.80)\end{array}$ & $\begin{array}{l}7.38 \pm 0.46 \\
(6.90-8.00)\end{array}$ & $12.73 \pm 2.74(9.40-15.60)$ & $8.93 \pm 1.67(6.70-10.30)$ & $2.80 \pm 0.94(2.00-4.00)$ \\
\hline Wmbu & $\begin{array}{c}20.60 \pm 1.71 \\
(19.20-23.10)\end{array}$ & $\begin{array}{l}7.53 \pm 0.52 \\
(6.90-8.00)\end{array}$ & $\begin{array}{c}33.58 \pm 9.53 \\
(19.40-39.90)\end{array}$ & $\begin{array}{c}22.90 \pm 5.61 \\
(14.60-26.30)\end{array}$ & $2.05 \pm 0.71(1.30-3.00)$ \\
\hline SPmak & $\begin{array}{c}19.03 \pm 1.52 \\
(17.30-21.00)\end{array}$ & $\begin{array}{l}6.98 \pm 0.64 \\
(6.50-7.90)\end{array}$ & $20.88 \pm 11.11(9.10-29.70)$ & $\begin{array}{l}16.03 \pm 6.47 \\
(9.90-22.10)\end{array}$ & $5.50 \pm 2.49(3.10-9.00)$ \\
\hline Tngo & $\begin{array}{c}20.78 \pm 2.25 \\
(18.00-23.10)\end{array}$ & $\begin{array}{l}7.18 \pm 0.49 \\
(6.70-7.80)\end{array}$ & $\begin{array}{c}22.925 \pm 8.045 \\
(15.40-32.10)\end{array}$ & $\begin{array}{c}15.73 \pm 5.13 \\
(10.90-21.80)\end{array}$ & $1.82 \pm 0.63(1.20-2.70)$ \\
\hline Wnja & $\begin{array}{c}21.63 \pm 1.43 \\
(20.00-23.30)\end{array}$ & $\begin{array}{l}6.40 \pm 0.14 \\
(6.30-6.60)\end{array}$ & $10.78 \pm 3.86(7.20-15.50)$ & $7.43 \pm 2.74(5.00-11.00)$ & $\begin{array}{c}2.018 \pm 1.027 \\
(0.77-3.00)\end{array}$ \\
\hline Wnjal & $\begin{array}{c}21.90 \pm 1.37 \\
(20.80-23.80)\end{array}$ & $\begin{array}{l}6.33 \pm 0.39 \\
(5.80-6.70)\end{array}$ & $21.78 \pm 8.70(13.00-32.10$ & $\begin{array}{c}15.10 \pm 5.57 \\
(9.50-21.80)\end{array}$ & $4.02 \pm 1.58(1.80-5.40)$ \\
\hline Tnk & $\begin{array}{c}20.13 \pm 1.19 \\
(19.00-21.80)\end{array}$ & $\begin{array}{l}7.20 \pm 0.29 \\
(6.80-7.50)\end{array}$ & $\begin{array}{c}30.58 \pm 12.99 \\
(17.20-48.3)\end{array}$ & $\begin{array}{c}20.60 \pm 7.76 \\
(12.20-30.90)\end{array}$ & $0.88 \pm 0.78(0.20-0.70)$ \\
\hline SPnke & $\begin{array}{c}20.20 \pm 1.57 \\
(18.00-21.60)\end{array}$ & $\begin{array}{l}6.88 \pm 0.49 \\
(6.30-7.50)\end{array}$ & $\begin{array}{l}24.73 \pm 10.70 \\
(10.20-26.50)\end{array}$ & $\begin{array}{c}16.90 \pm 7.11 \\
(7.30-24.40)\end{array}$ & $\begin{array}{c}6.88 \pm 2.96 \\
(3.10-10.00)\end{array}$ \\
\hline WHO & $10-25$ & $6.5-8.5$ & 2000 & 1000 & 5 \\
\hline
\end{tabular}

$\mathrm{T}$ = temperature; $\mathrm{pH}$ = hydrogen potential; $\mathrm{EC}=$ electrical conductivity; Tur = turbidity; $\mathrm{NTU}=$ nephelometric turbidity unit. Values presented are expressed as mean values \pm standard deviation (minimum-maximum) $(n=10)$.

TABLE 3: Results of analysed cations in the samples from ten water sources in Bangolan within the study period.

\begin{tabular}{|c|c|c|c|c|c|}
\hline \multirow{2}{*}{$\begin{array}{l}\text { Sample } \\
\text { codes }\end{array}$} & \multicolumn{5}{|c|}{ Parameters } \\
\hline & $\mathrm{Na}^{+}(\mathrm{mg} / \mathrm{L})$ & $\mathrm{K}^{+}(\mathrm{mg} / \mathrm{L})$ & $\mathrm{Ca}^{2+}(\mathrm{mg} / \mathrm{L})$ & $\mathrm{Mg}^{2+}(\mathrm{mg} / \mathrm{L})$ & $\mathrm{NH}_{4}^{+}(\mathrm{mg} / \mathrm{L})$ \\
\hline STnts & $1.52 \pm 1.23(0.32-3.21)$ & $\begin{array}{l}1.38 \pm 1.03 \\
(0.61-2.55)\end{array}$ & $\begin{array}{l}35.25 \pm 11.30 \\
(28.00-52.00)\end{array}$ & $6.08 \pm 2.40(4.20-9.30)$ & $\begin{array}{l}0.93 \pm 0.81 \\
(0.28-2.11)\end{array}$ \\
\hline Wnts & $0.95 \pm 0.51(0.51-1.67)$ & $\begin{array}{l}1.36 \pm 1.43 \\
(0.32-3.45)\end{array}$ & $\begin{array}{l}26.30 \pm 11.21 \\
(18.00-43.00)\end{array}$ & $\begin{array}{c}4.225 \pm 2.053 \\
(2.40-7.10)\end{array}$ & $\begin{array}{l}0.78 \pm 0.70 \\
(0.38-1.82)\end{array}$ \\
\hline Wnkw & $0.84 \pm 0.58(0.05-1.42)$ & $\begin{array}{l}1.80 \pm 0.52 \\
(1.02-2.11)\end{array}$ & $26.25 \pm 8.88(18.00-38.00)$ & $4.58 \pm 2.22(2.40-7.20)$ & $\begin{array}{c}0.67 \pm 1.01 \\
(0.05-2.18)\end{array}$ \\
\hline Wmbu & $0.62 \pm .38(0.06-0.91)$ & $\begin{array}{l}1.21 \pm 0.66 \\
(0.64-2.03)\end{array}$ & $23.5 \pm 7.0(15.00-31.00)$ & $3.03 \pm 2.83(0.60-7.10)$ & $\begin{array}{l}0.43 \pm 0.62 \\
(0.05-1.36)\end{array}$ \\
\hline SPmak & $1.35 \pm 1.46(0.40-3.50)$ & $\begin{array}{l}1.44 \pm 1.34 \\
(0.63-3.44)\end{array}$ & $21.25 \pm 7.72(14.00-32.00)$ & $4.45 \pm 2.45(2.90-8.10)$ & $\begin{array}{c}1.51 \pm 1.51 \\
(0.28-3.19)\end{array}$ \\
\hline Tngo & $1.10 \pm 0.84(0.04-1.83)$ & $\begin{array}{l}3.38 \pm 1.40 \\
(1.03-4.04)\end{array}$ & $\begin{array}{c}31.00 \pm 12.19 \\
(19.00-48.00)\end{array}$ & $3.93 \pm 2.89(1.80-8.10)$ & $\begin{array}{l}1.62 \pm 1.55 \\
(0.56-3.91)\end{array}$ \\
\hline Wnja & $0.38 \pm 0.37(0.04-0.77)$ & $\begin{array}{l}1.30 \pm 0.66 \\
(0.56-2.13)\end{array}$ & $22.00 \pm 6.48(14.00-29.00)$ & $4.80 \pm 3.50(2.40-10.00)$ & $\begin{array}{l}1.09 \pm 0.75 \\
(0.56-2.15)\end{array}$ \\
\hline Wnjal & $0.36 \pm 0.37(0.07-0.88)$ & $\begin{array}{l}1.44 \pm 1.12 \\
(0.63-3.10)\end{array}$ & $\begin{array}{c}22.75 \pm 11.12 \\
(14.00-39.00)\end{array}$ & $4.30 \pm 2.86(2.30-8.40)$ & $\begin{array}{l}0.92 \pm 0.24 \\
(0.64-1.15)\end{array}$ \\
\hline Tnk & $1.07 \pm 1.11(0.02-2.61)$ & $\begin{array}{l}1.70 \pm 0.76 \\
(1.03-2.41)\end{array}$ & $\begin{array}{l}43.00 \pm 13.32 \\
(32.00-62.00)\end{array}$ & $8.07 \pm 3.94(5.50-12.60)$ & $0.89 \pm 0.82(0.232 .08)$ \\
\hline SPnke & $\begin{array}{c}1.37 \pm 2.06 \\
(40.04-4.42)\end{array}$ & $\begin{array}{l}2.68 \pm 1.39 \\
(1.03-4.35)\end{array}$ & $22.25 \pm 9.54(14.00-36.00)$ & $10.05 \pm 5.51(4.80-17.8)$ & $\begin{array}{l}1.77 \pm 2.09 \\
(0.20-4.74)\end{array}$ \\
\hline WHO & 200 & 200 & 75 & 30 & 250 \\
\hline
\end{tabular}

Values presented are expressed as mean values \pm standard deviation (minimum-maximum) $(n=10)$.

and Shigella spp. Mean colony counts of Enterobacteria spp. ranged from $51.25 \pm 21.75 \mathrm{CFU} / 100 \mathrm{~mL}$ (Tngo) to $252.50 \pm 108.13 \mathrm{CFU} / 100 \mathrm{~mL}$. As shown on Figure 3 , the highest contamination level was recorded in July 2018, with STnts being the most contaminated (400 CFU/ $100 \mathrm{~mL}$ ). Mean colony counts of Escherichia coli ranged from $\quad 35.00 \pm 21.75 \mathrm{CFU} / 100 \mathrm{~mL}$ (Wnja) to $118.8 \pm 90.4 \mathrm{CFU} / 100 \mathrm{~mL}$ (SPnke) with STnts and SPnke being the most contaminated sources $(250 \mathrm{CFU} / 100 \mathrm{~mL})$ in July 2018 (Figure 4). Mean colony counts of Streptococcus spp. ranged from $36.25 \pm 32.24 \mathrm{CFU} / 100 \mathrm{~mL}$ (STnts) to $138.75 \pm 175.14 \mathrm{CFU} / 100 \mathrm{~mL}$ (SPnke) with 
TABLE 4: Results of analysed anions in the samples from ten water sources in Bangolan within the study period.

\begin{tabular}{|c|c|c|c|c|c|}
\hline \multirow{2}{*}{$\begin{array}{l}\text { Sample } \\
\text { codes }\end{array}$} & \multicolumn{5}{|c|}{ Parameters } \\
\hline & $\mathrm{HCO}_{3}^{-}(\mathrm{mg} / \mathrm{L})$ & $\mathrm{SO}_{4}^{2-}(\mathrm{mg} / \mathrm{L})$ & $\mathrm{NO}_{3}^{-}(\mathrm{mg} / \mathrm{L})$ & $\mathrm{Cl}^{-}(\mathrm{mg} / \mathrm{L})$ & $\mathrm{PO}_{4}^{3-}(\mathrm{mg} / \mathrm{L})$ \\
\hline STnts & $22.00 \pm 12.04(10.65-38.25)$ & $\begin{array}{c}1.04 \pm 0.97 \\
(0.20-2.40)\end{array}$ & $1.13 \pm 0.62(0.56-1.96)$ & $\begin{array}{c}0.28 \pm 0.21 \\
(0.01-0.39)\end{array}$ & $\begin{array}{l}0.26 \pm 0.37 \\
(0.01-0.80)\end{array}$ \\
\hline Wnts & $21.15 \pm 10.21(10.5-31.02)$ & $\begin{array}{l}0.60 \pm 0.35 \\
(0.30-1.01)\end{array}$ & $\begin{array}{c}0.98 \pm 0.90 \\
(0.43 \pm 2.32)\end{array}$ & $\begin{array}{l}0.04 \pm 0.04 \\
(0.01-0.03)\end{array}$ & $\begin{array}{l}0.20 \pm 0.28 \\
(0.00-0.61)\end{array}$ \\
\hline Wnkw & $\begin{array}{c}21.273 \pm 14.099 \\
(10.36-41.81)\end{array}$ & $\begin{array}{l}0.35 \pm 0.35 \\
(0.16-0.87)\end{array}$ & $0.62 \pm 0.84(0.05-1.83)$ & $\begin{array}{l}0.22 \pm 0.26 \\
(0.07-0.61)\end{array}$ & $\begin{array}{l}0.02 \pm 0.02 \\
(0.00-0.04)\end{array}$ \\
\hline Wmbu & $14.79 \pm 10.44(6.41-30.03)$ & $\begin{array}{l}0.58 \pm 0.40 \\
(0.19-1.06)\end{array}$ & $1.01 \pm 1.47(0.05-3.16)$ & $\begin{array}{l}0.29 \pm 0.49 \\
(0.02-1.02)\end{array}$ & $\begin{array}{l}0.05 \pm 0.07 \\
(0.00-0.14)\end{array}$ \\
\hline SPmak & $10.74 \pm 6.59(5.66-20.23)$ & $\begin{array}{l}1.29 \pm 1.58 \\
(0.20-3.60)\end{array}$ & $2.24 \pm 2.32(0.56-5.67)$ & $\begin{array}{l}0.44 \pm 0.59 \\
(0.01-1.30)\end{array}$ & $\begin{array}{l}0.74 \pm 0.63 \\
(0.05-1.56)\end{array}$ \\
\hline Tngo & $12.50 \pm 2.46(10.42-15.41)$ & $\begin{array}{l}0.83 \pm 0.92 \\
(0.19-2.18)\end{array}$ & $1.53 \pm 1.12(0.84-3.20)$ & $\begin{array}{l}0.21 \pm 0.36 \\
(0.01-0.75)\end{array}$ & $0.45 \pm 0.83(0.00-1.67$ \\
\hline Wnja & $12.093 \pm 4.023(9.32-18.07)$ & $\begin{array}{l}0.89 \pm 0.89 \\
(0.24-2.19)\end{array}$ & $0.84 \pm 0.26(0.56-1.18)$ & $\begin{array}{l}0.04 \pm 0.04 \\
(0.00-0.09)\end{array}$ & $\begin{array}{l}0.57 \pm 0.97 \\
(0.01-2.02)\end{array}$ \\
\hline Wnjal & $14.45 \pm 4.84(10.31-19.86)$ & $\begin{array}{l}0.77 \pm 0.60 \\
(0.21-1.50)\end{array}$ & $0.76 \pm 0.36(0.37-1.19)$ & $\begin{array}{l}0.28 \pm 0.23 \\
(0.05-0.53)\end{array}$ & $\begin{array}{l}0.24 \pm 0.35 \\
(0.04-0.76)\end{array}$ \\
\hline Tnk & $43.03 \pm 15.73(31.61-65.45)$ & $\begin{array}{l}0.76 \pm 0.89 \\
(0.15-2.08)\end{array}$ & $1.40 \pm 0.69(0.76-2.02)$ & $\begin{array}{l}0.52 \pm 0.58 \\
(0.06-1.36)\end{array}$ & $\begin{array}{l}1.01 \pm 0.74 \\
(0.01-1.74)\end{array}$ \\
\hline SPnke & $26.74 \pm 6.45(24.30-31.36)$ & $\begin{array}{l}1.43 \pm 1.85 \\
(0.19-4.11)\end{array}$ & $1.74 \pm 1.66(0.56-4.18)$ & $\begin{array}{c}0.42 \pm 0.72 \\
(0.01-1.50)\end{array}$ & $\begin{array}{l}1.72 \pm 1.83 \\
(0.28-4.17)\end{array}$ \\
\hline WHO & 1000 & 250 & 50 & 250 & $\leq 5$ \\
\hline
\end{tabular}

Values presented are expressed as mean values \pm standard deviation (minimum-maximum) $(n=10)$.

TABLE 5: Results of analysed heavy metals in the samples from ten water sources in Bangolan within the study period.

\begin{tabular}{|c|c|c|c|c|c|}
\hline \multirow{2}{*}{ Sample codes } & \multicolumn{5}{|c|}{ Parameters } \\
\hline & $\mathrm{Fe}^{2+}(\mathrm{mg} / \mathrm{L})$ & $\mathrm{Zn}^{2+}(\mathrm{mg} / \mathrm{L})$ & $\mathrm{Pb}^{2+}(\mathrm{mg} / \mathrm{L})$ & $\mathrm{Al}^{3+}(\mathrm{mg} / \mathrm{L})$ & $\mathrm{Cu}^{2+}(\mathrm{mg} / \mathrm{L})$ \\
\hline STnts & $0.19 \pm 0.22(0.01-0.51)$ & $\begin{array}{c}1.24 \pm 0.86 \\
(0.59-2.50)\end{array}$ & 0.00 & $\begin{array}{c}0.22 \pm 0.11 \\
(0.08-0.34)\end{array}$ & $1.16 \pm 1.38(0.16-3.11)$ \\
\hline Wnts & $0.01 \pm 0.01(0.00-0.03)$ & $\begin{array}{c}0.72 \pm 0.12 \\
(0.54-0.83)\end{array}$ & 0.00 & $0.25 \pm 0.16(0.10-0.44)$ & $\begin{array}{c}0.48 \pm 0.62 \\
(0.02-1.34)\end{array}$ \\
\hline Wnkw & $0.07 \pm 0.08(0.00-0.16)$ & $\begin{array}{l}0.66 \pm 0.29 \\
(0.38-1.03)\end{array}$ & 0.00 & $\begin{array}{c}0.31 \pm 0.32 \\
(0.05-0.75)\end{array}$ & $\begin{array}{l}1.37 \pm 0.59 \\
(0.78-2.18)\end{array}$ \\
\hline Wmbu & $0.07 \pm 0.13(0.00-0.26)$ & $\begin{array}{c}0.65 \pm 0.09 \\
(0.56-0.75)\end{array}$ & 0.00 & $\begin{array}{l}0.39 \pm 0.34 \\
(0.10-0.88)\end{array}$ & $\begin{array}{c}0.33 \pm 0.43 \\
(0.08-0.97)\end{array}$ \\
\hline SPmak & $0.66 \pm 1.11(0.00-2.32)$ & $1.07 \pm 1.39(0.18-3.11)$ & 0.00 & $0.15 \pm 0.15(0.06-0.37)$ & $\begin{array}{l}0.53 \pm 0.84 \\
(0.06-1.78)\end{array}$ \\
\hline Tngo & $\begin{array}{c}1.590 \pm 1.001 \\
(0.97-3.08)\end{array}$ & $1.10 \pm 1.21(0.14-2.71)$ & 0.00 & $\begin{array}{l}0.20 \pm 0.29 \\
(0.05-0.63)\end{array}$ & $0.13 \pm 0.14(0.04-0.34)$ \\
\hline Wnja & $0.38 \pm 0.3(0.06-0.74)$ & $\begin{array}{c}0.55 \pm 0.34 \\
(0.08-0.78)\end{array}$ & 0.00 & $\begin{array}{c}0.19 \pm 0.28 \\
(0.03-0.60)\end{array}$ & $\begin{array}{c}0.31 \pm 0.44 \\
(0.04-0.96)\end{array}$ \\
\hline Wnjal & $0.44 \pm 0.35(0.12-0.78)$ & $0.73 \pm 0.50(0.14-1.36)$ & 0.00 & $0.14 \pm 0.14(0.06-0.34)$ & $\begin{array}{c}0.33 \pm 0.44 \\
(0.06-0.96)\end{array}$ \\
\hline Tnk & $0.85 \pm 0.68(0.20-1.50)$ & $\begin{array}{l}2.26 \pm 1.17 \\
(0.98-3.80)\end{array}$ & 0.00 & $\begin{array}{l}0.52 \pm 0.20 \\
(0.31-0.78)\end{array}$ & $1.09 \pm 0.81(0.17-2.14)$ \\
\hline SPnke & $1.76 \pm 1.03(0.87-3.24)$ & $1.77 \pm 1.15(0.18-2.89)$ & $\begin{array}{c}0.003 \pm 0.005 \\
(0.00-0.01)\end{array}$ & $\begin{array}{c}0.23 \pm 0.22 \\
(0.03-0.44)\end{array}$ & $\begin{array}{c}0.42 \pm 0.43 \\
(0.04-0.88)\end{array}$ \\
\hline WHO & - & 3 & 0.01 & 0.2 & 2 \\
\hline
\end{tabular}

Values presented are expressed as mean values \pm standard deviation (minimum-maximum) $(n=10)$.

STnts and Tngo being the least contaminated sources (5 CFU/100 mL) in April and July 2018, respectively, and SPnke the most contaminated (400 CFU/100 mL) in July 2018 (Figure 5). Mean colony counts of Salmonella spp. ranged from $8.75 \pm 8.54 \mathrm{CFU} / 100 \mathrm{~mL}$ (Tngo) to $93.8 \pm 42.7 \mathrm{CFU} / 100 \mathrm{~mL}$ (Wmbu) with the highest contamination levels recorded in July 2018 (Figure 6). Mean colony counts of Shigella spp. ranged from $1.3 \pm 2.5 \mathrm{CFU} / 100 \mathrm{~mL}$ (SPmak) to $16.25 \pm 22.87 \mathrm{CFU} /$ $100 \mathrm{~mL}$ (Wnja1). Shigella spp. was absent in most of the samples between November 2017 and January and April 2018 (Figure 7). 


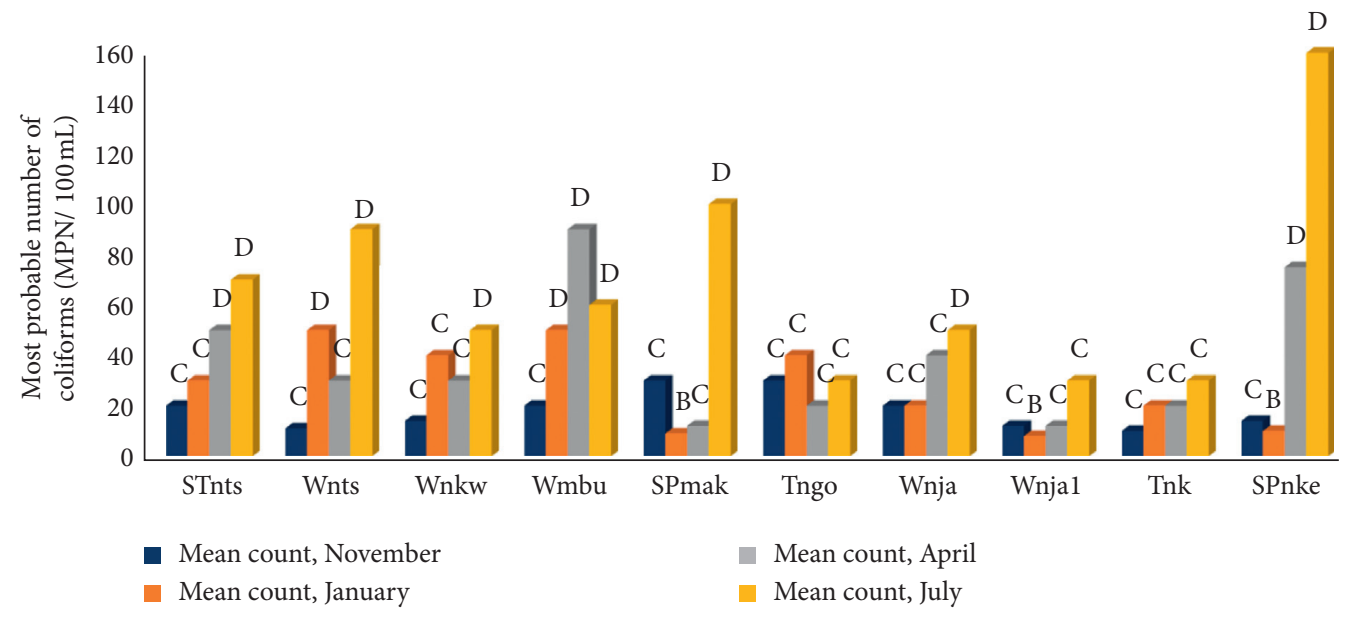

Figure 2: Most probable number of coliforms in $100 \mathrm{~mL}$ of water between November 2017 and July 2018. A= excellent; B = acceptable, low risk; $\mathrm{C}=$ unacceptable, high risk; $\mathrm{D}=$ grossly polluted.

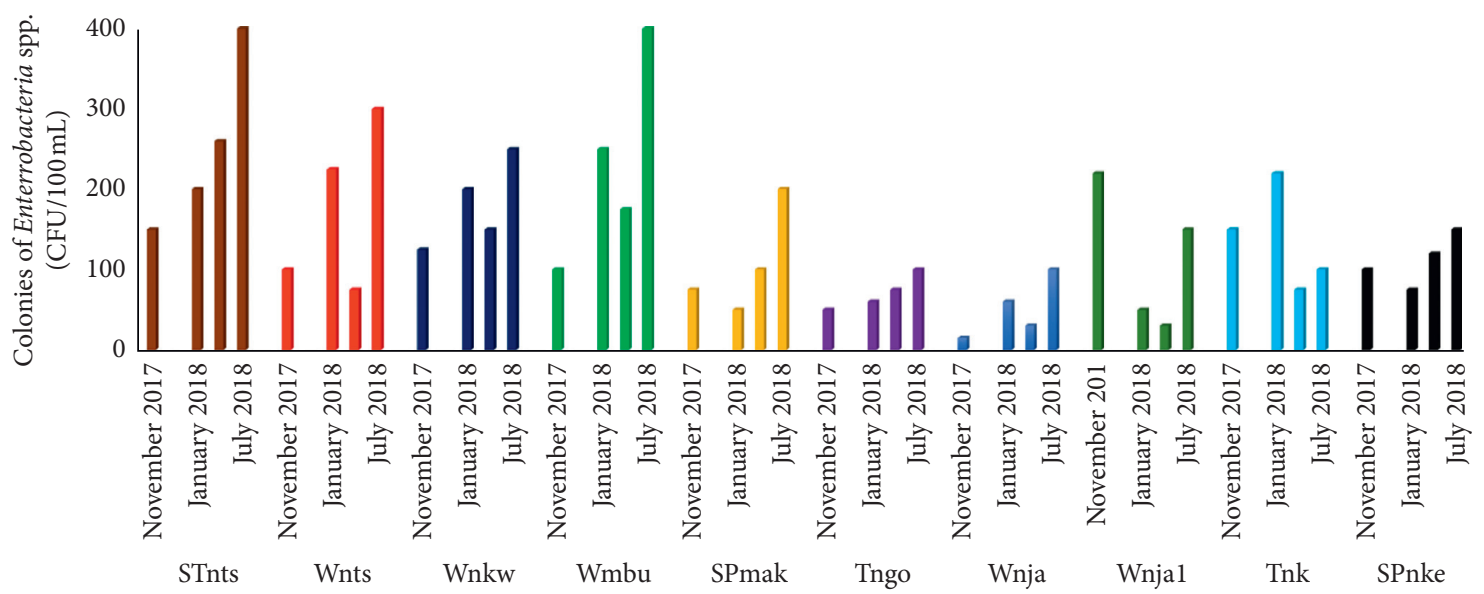

FIgURE 3: Colonies of Enterobacteria spp. isolated in the sampled waters between November 2017 and July 2018.

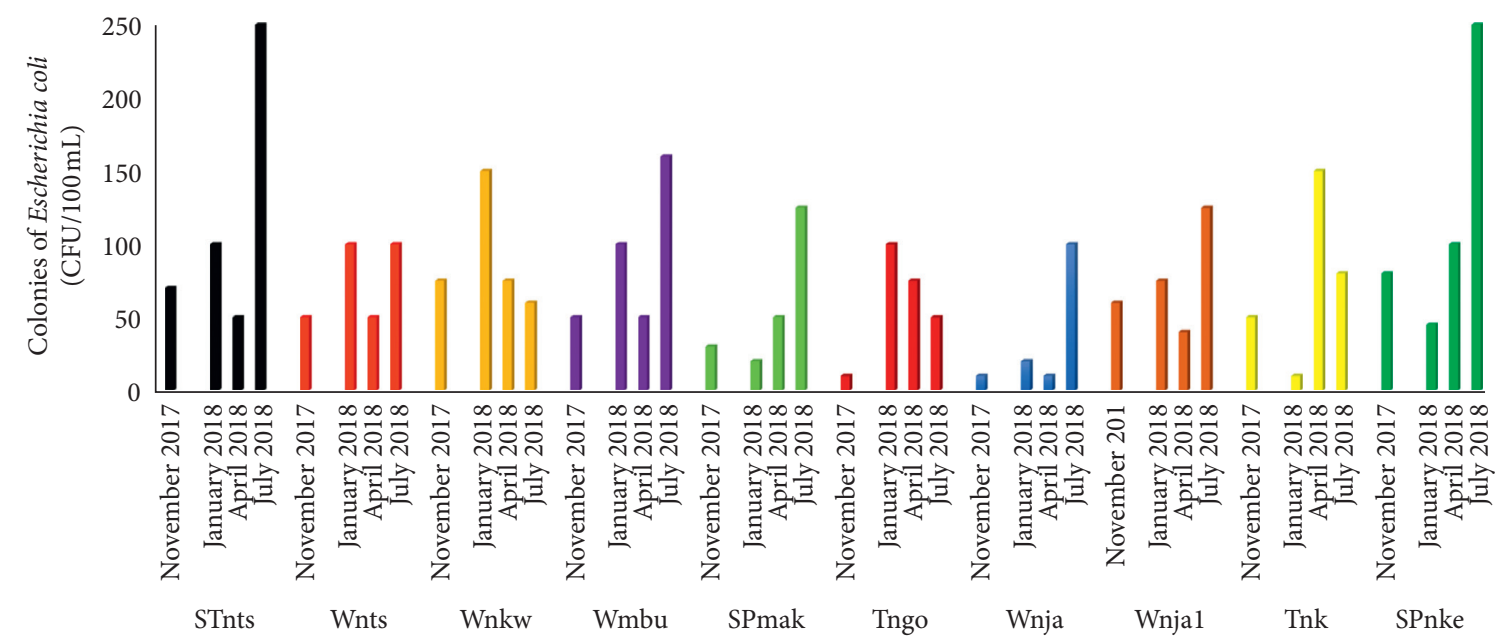

FIGURE 4: Colonies of Escherichia coli isolated in the sampled waters between November 2017 and July 2018. 


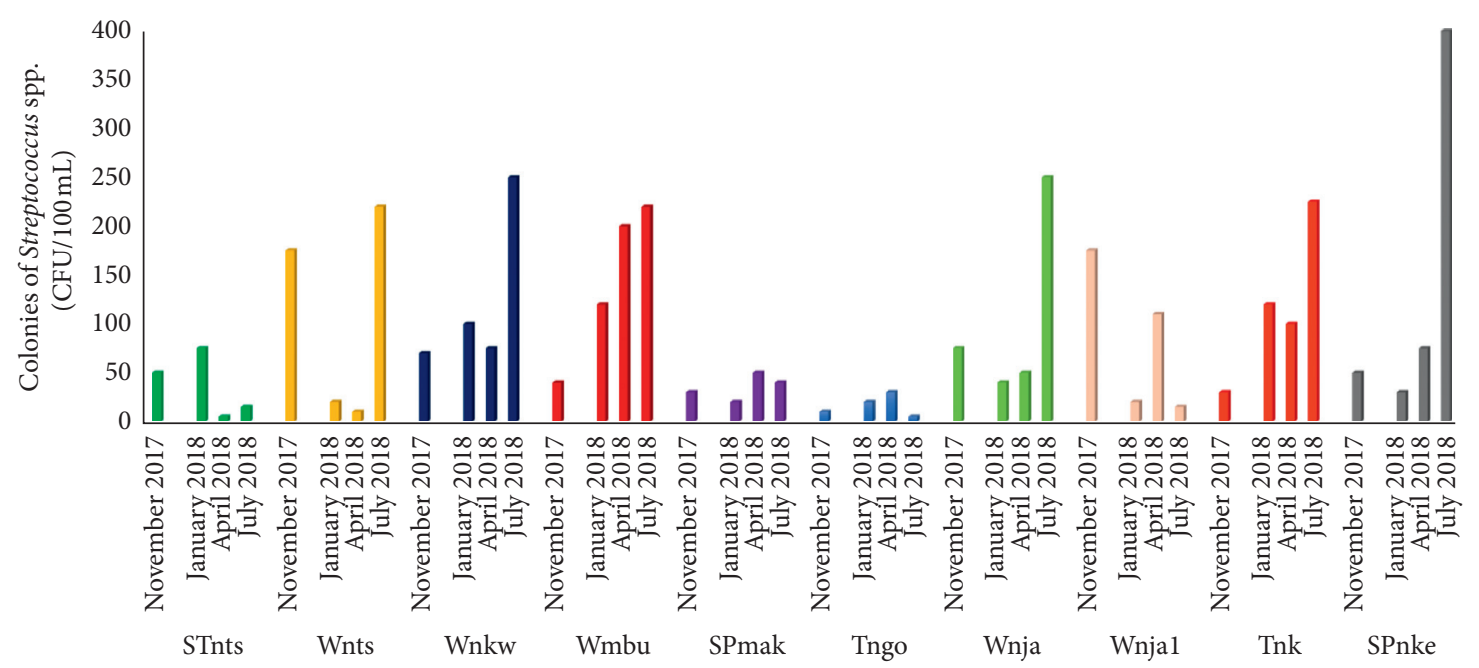

Figure 5: Colonies of Streptococcus spp. isolated in the sampled waters between November 2017 and July 2018.

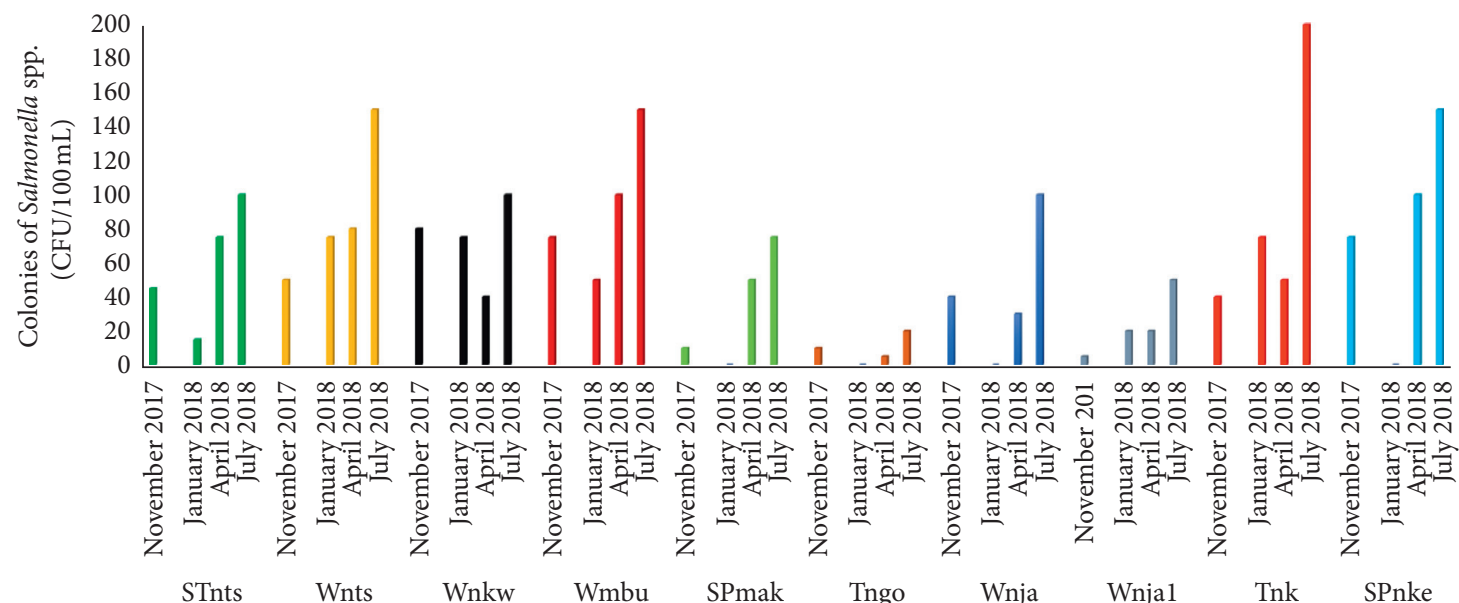

Figure 6: Colonies of Salmonella spp. isolated in the sampled waters between November 2017 and July 2018.

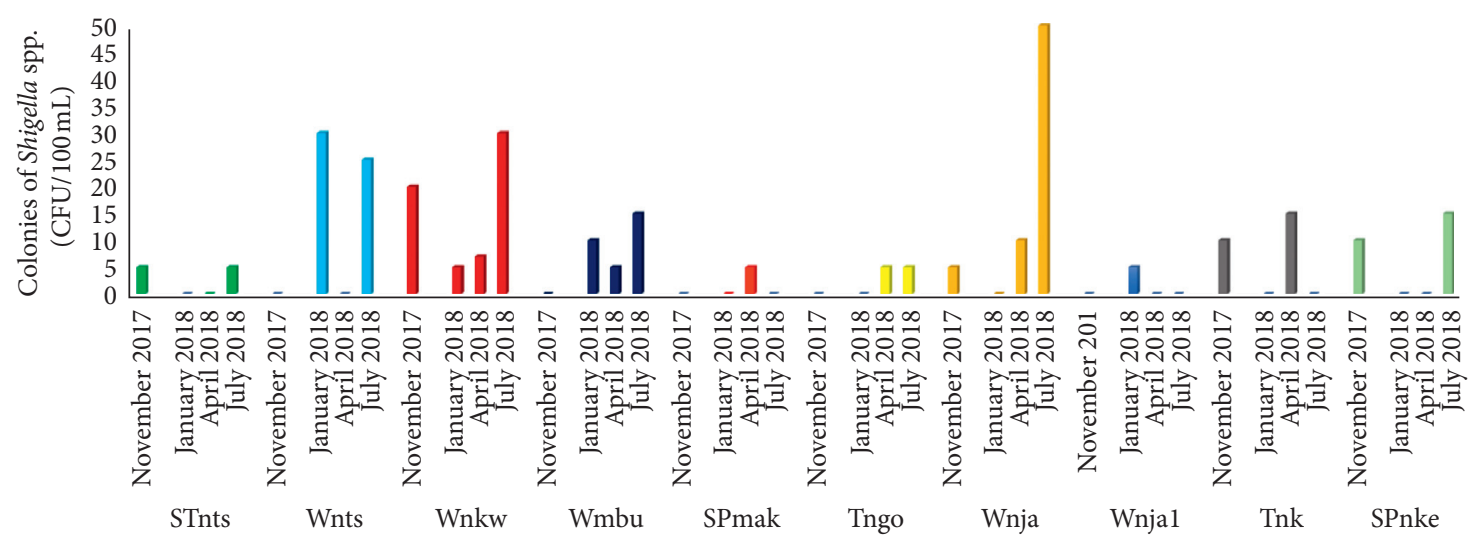

Figure 7: Colonies of Shigella spp. isolated in the sampled waters between November 2017 and July 2018.

\subsection{Discussion}

3.2.1. Physical Parameters. Temperature values were between the minimum and maximum annual temperatures in this area, also falling within the WHO guideline range of 15 to $25^{\circ} \mathrm{C}$. ANOVA revealed a significant difference between temperature mean values in all the sampling seasons $(p<0.001)$, and the post hoc comparisons using the Tukey HSD test further indicated that the difference was between the mean temperature in July and the other 


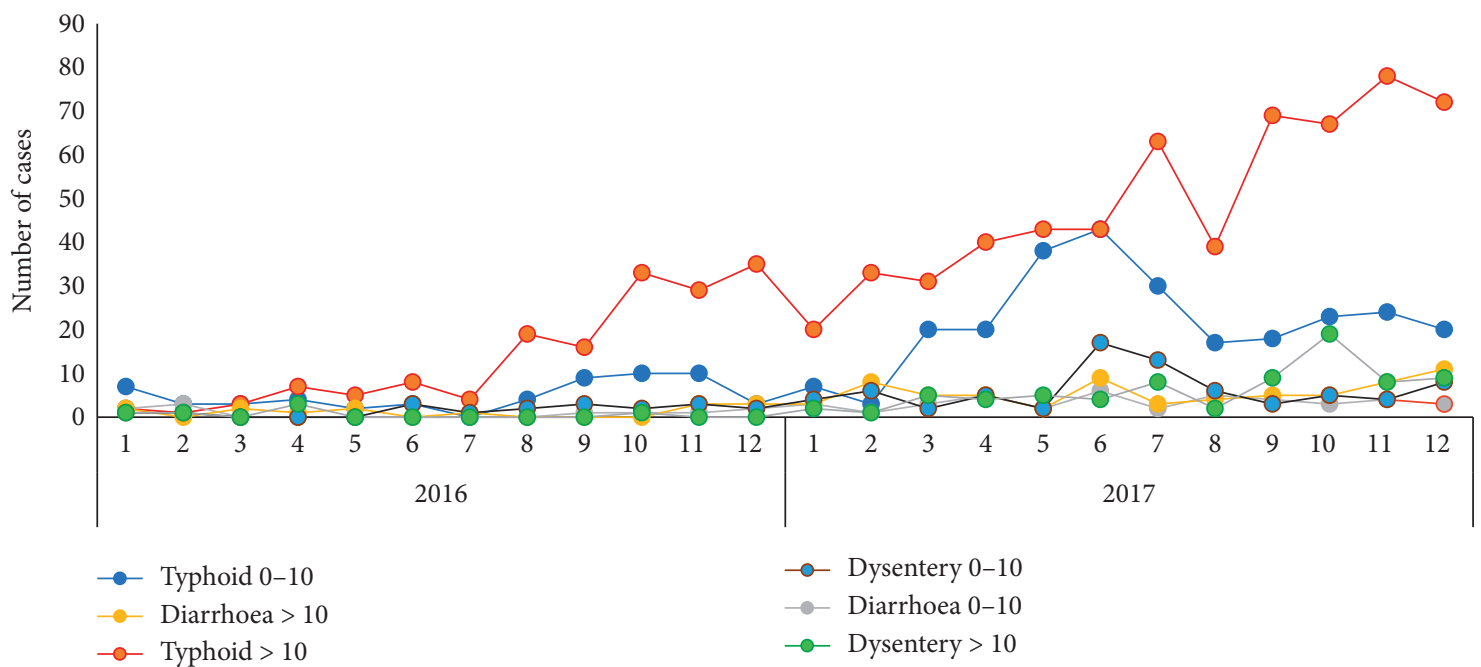

FIGURE 8: Monthly distribution of waterborne diseases recorded in Bangolan between 2016 and 2017.

months. This was a significant increase, which could be as the result of seasonal and daily changes, shade, air temperature, water depths, and inflow of the groundwater [6]. Similar temperature values were obtained by Wirmvem et al. [19], while studying the shallow groundwater recharge mechanism and apparent age in the Ndop plain, Northwest Cameroon. Looking at the health aspect, the values were within the permissible range. But it should be noted that high water temperature enhances the growth of microorganisms and may increase problems related to taste, odour, colour, and corrosion [8]. $\mathrm{pH}$ did not vary significantly with seasons $(p>0.05)$, and the water sources were found to range from moderately acidic to weakly basic. Wnts and Wnja had relatively low $\mathrm{pH}$ values compared to the WHO permissible range $(6.5-8.5)$ in most of the sampling seasons, as seen in Table 3. The $\mathrm{pH}$ of the ground water sources in January was in disagreement with those reported by Wirmvem et al. [20] in the same area. Lower mean $\mathrm{pH}$ observed in April and July could be as a result of acid rain [6]. Low EC and TDS in the water sources suggests low mineralization and freshwaters. The strong positive correlation observed between EC and TDS $(r=1, p<0.001)$ could be justified by the fact that EC arises from dissolved ionic matter. Similar $\mathrm{pH}$ and low electrical conductivity have also been reported elsewhere in Cameroon [5, 21]. Wu et al. [22] reported relatively high pH and TDS in Beijiao Water Source of Yinchuan in China. Variations in turbidity was significant with seasons $(p<0.05)$, and the statistical difference was found to be between the mean value in July and the other months. Based on the results, STnts, SPmak, Wnja1, and SPnke sources had turbidity values above the WHO guideline value of 5 NTU. High turbidity in July could be due to heavy rains. This indicates the possible presence of hazardous chemical and microbial contaminants [8]. High turbidity associated with iron concentrations was also reported in water sources from the Sourou Valley in Burkina Faso [23].
3.2.2. Chemical Parameters. The presence of calcium and magnesium ions in the studied water sources suggests the occurrence of limestone and chalk sediments in the study area [6]. Looking at the concentrations (Table 3), calciumbased hardness was predominating due to the fact that magnesium is usually found at lower concentrations in groundwater [24]. Increase in both ions concentration in July 2018 was significant $(p<0.001)$, and this could be attributed to runoff from nearby soils. Calcium and magnesium concentrations were in accordance with those of Tamungang et al. [21] but disagreed with the findings of Emile [25] who reported very low calcium and magnesium in drinking water from alternative sources in the Dschang Municipality, in the West region of Cameroon. $\mathrm{Na}^{+}, \mathrm{K}^{+}$, and $\mathrm{Cl}^{-}$concentrations were also very low compared to WHO standards of $200 \mathrm{mg} / \mathrm{L}$ for $\mathrm{Na}^{+}$and $\mathrm{K}^{+}$and $250 \mathrm{mg} / \mathrm{L}$ for $\mathrm{Cl}^{-}$, respectively. Low sodium and chloride ions in the sampled waters could be as a result of low $\mathrm{NaCl}$ in the geological formations of the study area, as both ions are generally from the decomposition of rock salts like sodium and aluminium silicates [5, 26]. According to Wirmvem et al., [20], low $\mathrm{K}^{+}$ may be due to its low geochemical mobility in the area. Bicarbonate was the only ion responsible for alkalinity as $\mathrm{CO}_{3}^{2-}$ and $\mathrm{OH}^{-}$were absent. Its concentrations were very low compared to the WHO guideline value of $1000 \mathrm{mg} / \mathrm{L}$. The presence of $\mathrm{HCO}_{3}^{-}$was necessary as it constitutes an important buffer system which helps in lowering the acidity of water [27]. A significant difference $(p<0.01)$ was observed in its concentrations between the sampling sessions. $\mathrm{HCO}_{3}^{-}$originates from the partitioning of $\mathrm{CO}_{2}$ in the atmosphere and the weathering of carbonate minerals in rocks such as limestone and dolomite. Wirmwem et al. [20] also reported low $\mathrm{HCO}_{3}^{-}$in some bore holes and wells in this area which was associated with the possible presence of igneous rocks. Sulphate concentrations were insignificant regarding the WHO guideline value of $250 \mathrm{mg} / \mathrm{L}$. Low sulphates suggest low and possible absence of evaporitic sedimentary rocks such as gypsum $\left(\mathrm{CaSO}_{4}\right)$ and pyrite $(\mathrm{FeS})$ in the study 
area, and the significant increase in its concentrations in July $(p<0.001)$ was surely due to the effect of rain and the use of fertiliser and detergents [28]. Nitrates found in the sampled waters in January probably came from nitrate producing bacteria, and the significant increase observed in its concentrations in July $(p<0.001)$ suggests its infiltration from waste discharges and fertilisers into the water bodies [8]. However, these concentrations were very low compared to the guideline value of $50 \mathrm{mg} / \mathrm{L}$. Interest is centred on nitrates mostly because high nitrate levels in water have been reported to be responsible for the "blue baby" syndrome (methaemoglobinaemia) and typhoid effects [8]. Low sodium and potassium and high sulphates and nitrates have been reported elsewhere in Cameroon [29]. Lagnika et al. [30] reported sulphate levels between 0.5 and $26 \mathrm{mg} / \mathrm{L}$ and very high nitrates above the WHO limit in the wells of Pobè municipality in Benin, associated with agricultural practices and the proximity of septic tanks. $\mathrm{NH}_{4}^{+}$found in the water sources was surely from biological breakdown of domestic and agricultural wastes, and its presence was thus an indicator of bacterial, sewage, and animal wastes contaminations $[6,31]$. However, its low concentrations far below the permissible limit of $30 \mathrm{mg} / \mathrm{L}$ prescribed by the WHO suggested no associated health risk. Phosphate concentrations were also very low in all the sampled waters without any significant differences with seasons. This could be due to its sorption on organic colloids. This is in accordance with the results of Wirmvem et al. [20] who also reported the absence of phosphate in groundwater in this area.

3.2.3. Heavy Metals. Lead was below detectable limits in all the samples except in SPnke in July (Table 5). The quasiabsence of lead could be justified by the fact that it generally comes from galvanized iron pipe plumbing [6]. Hence, there was no risk of lead intoxication. Olusola et al. [32] reported high lead above the WHO permissible limits in ground water in Southwest Nigeria which was associated with human activities. Iron was low in most of the water sources and had concentrations above $1 \mathrm{mg} / \mathrm{L}$ in Tngo, Tnk, and SPnke in most of the sampling sessions. Although iron is an essential element in human nutrition, its presence in domestic water is a burden as it promotes the growth of "iron bacteria," which derive their energy from the oxidation of ferrous iron to ferric iron and, in the process, deposit a slimy coating on the piping. At levels above $0.3 \mathrm{mg} / \mathrm{L}$, it stains laundry and plumbing fixtures and develops noticeable taste in water. But no health-based guideline value has been proposed for this element [8]. Iron in these water sources surely came from nearby soils as industrial activities are absent in this locality. A similar observation was made by Tamungang et al. [27] in the Bagangte municipality, West Cameroon. In contrast, Njoyim et al. [4] reported very high lead and iron in Bambui's community drinking water found in the same region. Zinc was below the WHO guideline value of $3 \mathrm{mg} / \mathrm{L}$ for drinking water in nearly all the water sources during all the sampling sessions. Its presence could be as a result of its leaching from soils and the corrosion of galvanized materials for the transport of tap water [33]. Zinc is an essential trace element that is a catalytic component of over 300 enzymes, which also has a role in the structural integrity of proteins and membranes, in the union of hormones to its receptors and in gene expression. It is required for growth, normal development, DNA synthesis, immunity, and sensory functions. The major problem associated with its concentrations in domestic water lies on the acceptability aspect due to the unpleasant taste of water containing it above $3 \mathrm{mg} / \mathrm{L}[6,33]$. Copper levels were insignificant compared with the WHO guideline value of $2 \mathrm{mg} / \mathrm{L}$ except in Wnkw in November with a concentration of $2.18 \mathrm{mg} / \mathrm{L}$ and STnts with a concentration of $3.11 \mathrm{mg} / \mathrm{L}$ in July. Even though copper is responsible for structural and catalytic properties of multiple enzymes necessary for normal body functions and also necessary for infant growth, host defense mechanisms, bone strength, red and white cell maturation, iron transport, and brain development, it is responsible for the metallic taste of water and a green staining of clothing, fixtures, and hair at concentrations above $2 \mathrm{mg} / \mathrm{L}[6,34]$. Low copper in these water sources maybe due to its low content in the soils of the study area and also the fact that the current water $\mathrm{pH}$ did not favour its dissolution [8]. Khan [35] also reported low copper, lead, zinc, and iron in drinking water in Aligarh, India. Aluminium was also found in low concentrations in all the sources and mostly exceeded the WHO guideline value of $0.2 \mathrm{mg} / \mathrm{L}$ in July 2018 but without exceeding $1 \mathrm{mg} / \mathrm{L}$. Its low content in the studied water was surely due to the fact that water $\mathrm{pH}$ did not favour it dissolution from soils. Though not originally considered to be a significant health hazard in drinking water, aluminium has more recently been shown to pose a danger to persons suffering from kidney disorders. It is also responsible for neurological problems and has been cited as a contributory factor to Alzheimer disease [36]. Apart from zinc which did not vary significantly with season $(p>0.444)$, ANOVA revealed a significant difference in aluminium and copper contents at $p<0.001$ and iron at $p<0.048$ levels. Post hoc comparisons using the Tukey HSD test indicated that the difference was between the concentrations in July and the other months. This was a significant increase in concentrations which was surely due to the effects of the rains. The positive correlation observed between Fe and $\mathrm{Zn}(r=0.772)$, $\mathrm{Zn}$ and $\mathrm{Al}(r=0.650), \mathrm{Mg}$ and $\mathrm{Fe}(r=0.786), \mathrm{Mg}$ and $\mathrm{Zn}$ $(r=0.854), \mathrm{Mg}$ and $\mathrm{Al}(r=0.649), \mathrm{K}$ and $\mathrm{Fe}(r=0.850), \mathrm{K}$ and $\mathrm{Ca}(r=0.720)$, and $\mathrm{K}$ and $\mathrm{Mg}(r=0.713)$ could be due to their common origin.

3.2.4. Bacteriological Quality. The presence of faecal coliforms (Figure 2) suggests recent contamination of the water sources by human or animal faeces and the possible presence of other pathogenic organisms $[18,21]$. This was confirmed through the identification of specific bacteria, namely, Enterobacteria spp., Escherichia coli, Streptococcus spp., Salmonella spp., and Shigella spp. (Figures 3-7). Based on the WHO guideline that recommends no bacteria of faecal origin in drinking water, all the sources were unfit for domestic uses such as drinking and bathing. Similar high faecal coliform counts have also been reported in other parts 
of Cameroon by Katte et al. [11], Mofor et al. [5], Nanfack et al. [18], Njoyim et al. [4], Tamungang et al. [27], Emile [25], and elsewhere in Africa [23, 26, 37]. Nogueira et al. [38] also reported high faecal coliforms in $83 \%$ of untreated water sources in Brazil. According to Kuhn et al. [34], domestic water with faecal coliform content above $100 \mathrm{CFU} / 100 \mathrm{~mL}$ will lead to serious health effects if used for drinking and cooking and will possibly cause infections if used for bathing and laundry. The presence of faecal coliforms and the abundance of specific pathogenic bacteria in the studied water sources can be associated with poor hygiene and sanitation. The springs and stream were located in areas with free access to animals and were not properly taken care of. From observations made in the field, mostly children were found using unclean containers to carry water from the springs and some adults were also found doing laundry around the sources which, according to Nanfack et al. [18], had strong pathological impacts on the water sources. In addition, the enormous pollution of these water sources could be explained using other factors amongst which are those related to the environment such as floods in the rainy season, infiltration of organic matter in the soil, the low depth of the groundwater table, and the behaviour of the population through open-air defecation. These partly explain the contamination of tap water as the spring sources of the main catchment are found in a valley, and during the rainy season, mud, leaves, and other foreign objects are introduced into the catchment [14]. The high occurrence of faecal coliforms and other pathogenic bacteria in the wells may be due to the construction of the wells near septic tanks [39]. High colonies of pathogenic bacteria were also reported in domestic waters in the neighbouring Babessi village by Tamungang et al. [21]. The significant increase in the colony counts of most of the isolated bacteria in July 2018 could be due to the effects of rain, and the positive correlations between them justified their common origin.

The population of the study area often judged their water for domestic uses based on organoleptic parameters such as appearance, taste, and odour. However, this exposes them to water-related diseases such as typhoid, diarrhoea, and dysentery, given the high colonies of pathogenic bacteria in these sources. Data obtained from health centres in the locality on water-borne diseases between 2016 and 2017 confirmed the prevalence of typhoid to which were also associated diarrhoea and dysentery, as shown in Figure 8. A total of 1389 cases of these diseases were recorded during this period. It is seen from Figure 8 that the number of cases were increasing from 2016 to 2017 . The data showed that 465 of the reported cases were children below the age of 10 years and the remaining majority had ages above 10 years. This was in contradiction with the prediction of WHO which states that children below 5 years are the most exposed to water-borne diseases. It should be noted that these were just few cases out of many, as many people in this locality preferred traditional medicine for the treatment of these diseases rather than going to the hospital, observation shared by Nanfack et al. [18]. Also, others may prefer going to bigger hospitals not far from the locality for better health care. The water sources were of bad quality and thus not suitable for drinking and bathing but had insignificant effect on laundry and cooking.

\section{Conclusion}

This study focused on the quality of domestic water sources in Bangolan, Northwest Cameroon, based on the WHO guidelines. The results of physical parameters showed that all the water sources had temperatures within the WHO acceptable limits and the $\mathrm{pH}$ ranged from moderately acidic to weakly basic with very low mineral content. The results of chemical parameters indicated that the samples had below average concentrations of nitrates, phosphates, sulphates, and bicarbonates. The results of heavy metals and mineral elements indicated that iron and aluminium were found above the WHO guideline values in most seasons, and this was attributed to the nature of the soils in the locality. The results of bacteriological analyses indicated that faecal coliforms and other specific bacteria were also found in the sampled waters, suggesting recent contamination of the sources by human or animal faeces. Statistical analyses showed that heavy rain had significant influence on water quality. Prevalence of water-borne diseases in the locality was and still remains a major public health concern. Given the poor bacteriological quality of the water sources, home treatment methods such as chlorination, filtration, boiling, and solar disinfection should be implemented prior to consumption.

\section{Data Availability}

Relevant data are available for consultation if needed.

\section{Conflicts of Interest}

The authors declare that there are no conflicts of interest regarding the publication of this article.

\section{Acknowledgments}

We thank the health authorities of Bangolan for providing us with the necessary information needed to accomplish this research. We also thank the Babessi council for the financial support offered.

\section{References}

[1] D. A. Shigut, G. Liknew, D. D. Irge, and T. Ahmad, “Assessment of physico-chemical quality of borehole and spring water sources supplied to Robe Town, Oromia region, Ethiopia," Applied Water Science, vol. 7, no. 1, pp. 155-164, 2016.

[2] United Nations International Children Emergency Fund (UNICEF), UNICEF Handbook on Water Quality, United Nations International Children Emergency Fund (UNICEF), New York, NY, USA, 2008.

[3] World Health Organization (WHO), Guidelines for DrinkingWater Quality, WHO, Geneva, Switzerland, 2010.

[4] E. B. T. Njoyim, N. A. Mofor, M. L. F. Nib, and J. Sunjo, "Physicochemical and bacteriological quality assessment of the Bambui community drinking water in the North West 
Region of Cameroon," African Journal of Environmental Science and Technology, vol. 10, no. 6, pp. 181-191, 2016.

[5] N. A. Mofor, E. B. T. Njoyim, and A. D. Mvondo-Zé, "Quality assessment of some springs in the awing community, Northwest Cameroon, and their health implications," Journal of Chemistry, vol. 2017, Article ID 3546163, 11 pages, 2017.

[6] World Health Organization (WHO), Guidelines for DrinkingWater Quality, WHO Library Cataloguing-in-Publication Data, Geneva, Switzerland, 4th edition, 2011.

[7] World Health Organization (WHO) \& United Nations International Children Emergency Fund (UNICEF), Progress on Sanitation and Drinking Water, 2015 Update and MDG Assessment Report, World Health Organization \& United Nations International Children Emergency Fund, Geneva, Switzerland, 2015.

[8] World Health Organization (WHO), Guidelines for DrinkingWater Quality, Incorporating the First Addendum, WHO Library Cataloguing-in-Publication Data, Geneva, Switzerland, 4th edition, 2017.

[9] The United Nations (UN), Transforming Our World: The 2030 Agenda for Sustainable Development, United Nations (UN), New York, NY, USA, 2015.

[10] A. A. Ako, G. E. T. Eyong, and G. E. Nkeng, "Water resources management and integrated water resources management (IWRM) in Cameroon," Water Resources Management, vol. 24 , no. 5, pp. 871-888, 2010.

[11] V. Y. Katte, M. F. Fonteh, and G. N. Guemuh, "Domestic water quality in urban centres in Cameroon: a case study of Dschang in the West Province," African Water Journal, pp. 43-54, 2003.

[12] M. J. Wirmvem, W. Y. Fantong, E. R. Wotany, T. Ohba, and S. Ayonghe, "Sources of bacteriological contamination of shallow groundwater and health effects in Ndop plain, Northwest Cameroon," Journal of Environmental Science and Water Resources, vol. 2, no. 4, pp. 127-132, 2013.

[13] Field data obtained from the bangolan intergrated health centre, 2017.

[14] Cameroon Association of Active Youths (CAMAAY), "Study on water management in four villages of Babessi municipality," A Survey Report, Cameroon Association of Active Youths, Bamenda, Cameroon, 2015.

[15] United Councils and Cities of Cameroon (UCCC), Babessi council, 2019, http://www.cdr-cvuc.cm/index.php/fr/ Mozilla_firefox.

[16] J. Rodier, B. Legube, and N. Merlet, L'analyse de l'eau, eaux Naturelles, eaux Résiduaires, eau de mer: Chimie, Physicochimie, Microbiologie, Biologie, Interprétation des Résultats, Editions Dunod, Paris, France, 9eme edition, 2009.

[17] World Health Organization (WHO), Water and Sanitation Related Diseases Fact, WHO Library Cataloguing-in-Publication Data, Geneva, Switzerland, 2006.

[18] N. A. C. Nanfack, F. A. Fonteh, V. K. Payne, B. Katte, and J. M. Fogoh, "Eaux non conventionnelles: un risque ou une solution aux problèmes d'eau pour les classes pauvres," Larhyss Journal, vol. 17, pp. 47-64, 2014.

[19] M. J. Wirmvem, M. E. Mimba, B. T. Kamtchueng et al., "Shallow groundwater recharge mechanism and apparent age in the Ndop plain, Northwest Cameroon," Applied Water Science, vol. 7, no. 1, pp. 489-502, 2014.

[20] M. J. Wirmvem, T. Ohba, W. Y. Fantong et al., "Hydrochemistry of shallow groundwater and surface water in the Ndop plain, North West Cameroon," African Journal of Environmental Science and Technology, vol. 7, no. 6, pp. 518-530, 2013.
[21] N. E. B. Tamungang, N. F. Biosengazeh, M. N. Alakeh, and D. Y. Tameu, "Contrôle de la qualité des eaux domestiques dans le village Babessi au Nord-Ouest Cameroun," International Journal of Biological and Chememical Sciences, vol. 10, no. 3, pp. 1382-1402, 2016.

[22] H. Wu, J. Chen, H. Qian, and X. Zhang, "Chemical characteristics and quality assessment of groundwater of exploited aquifers in Beijiao water source of Yinchuan, China: a case study for drinking, irrigation, and industrial purposes," Journal of Chemistry, vol. 2015, Article ID 726340, 14 pages, 2015.

[23] S. Boubacar, K. Aminata, Z. Dramane et al., "Problematic of drinking water access in rural area: case study of the Sourou Valley in Burkina Faso," Journal of Environmental Protection, vol. 4, no. 1, pp. 31-50, 2013.

[24] National Research Council (NRC), Drinking Water and Health, National Academy of Sciences, Washington, DC, USA, 1977.

[25] T. Emile, "Chemical and bacteriological analysis of drinking water from alternative sources in the Dschang municipality, Cameroon," Journal of Environmental Protection, vol. 2, no. 5, pp. 620-628, 2011.

[26] M. L. Belghiti, A. Chahlaoui, D. Bengoumi, and R. El Moustaine, "Etude de la qualite physico-chimique et Bactériologique des eaux souterraines de la nappe plio-quaternaire dans la région de meknés (Maroc)," Larhyss Journal, vol. 14, pp. 21-36, 2013.

[27] N. E. B. Tamungang, T. R. Menga, N. A. Mofor, F. B. Nchofua, and I. K. Njoyim, "Evaluation of surface and ground water quality in the Bangangte municipality-West Cameroon," International Journal of Research and Review in Applied Sciences, vol. 28, no. 2, pp. 53-64, 2016.

[28] D. Ghazali and A. Zaid, "Etude de la qualite physico-chimique et bacteriologique des eaux de la source ain salama-jerri (region de meknes-maroc)," Larhyss Journal, vol. 12, pp. 25-36, 2013.

[29] A. A. Ako, G. E. T. Eyong, J. Shimada et al., "Nitrate contamination of groundwater in two areas of the Cameroon volcanic line (banana plain and mount Cameroon area)," Applied Water Science, vol. 4, no. 2, pp. 99-113, 2013.

[30] M. Lagnika, M. Ibikounle, J. C. Montcho, V. D. Wotto, and N. G. Sakiti, "Caractéristiques physico-chimiques de l'eau des puits dans la commune de Pobè (Bénin, Afrique de l'ouest)," Journal of Applied Biosciences, vol. 79, no. 1, pp. 6887-6897, 2014.

[31] A. Akil, T. Hassan, E. H. Fatima, B. Lahcen, and L. Abderrahim, "Etude de la qualité physico-chimique et contamination métallique des eaux de surface du bassin versant de guigou, maroc," European Scientific Journal, vol. 10, no. 23, pp. 1857-7881, 2014.

[32] A. Olusola, O. Adeyeye, and O. Durowoju, "Groundwater: quality levels and human exposure, SW Nigeria," Journal of Environmental Geography, vol. 10, no. 1-2, pp. 23-29, 2017.

[33] World Health Organization (WHO), Nutrients in Drinking Water, WHO Library Cataloguing-in-Publication Data, Geneva, Switzerland, 2005.

[34] A. Kuhn, W. N. Lesufi, and A. P. M. Oelofse, Quality of Domestic Water Supply Volume One: Assessment Guide, Water Research Commission, Pretoria, South Africa, 2001.

[35] T. A. Khan, "Trace elements in the drinking water and their possible health effects in Aligarh city, India," Journal of Water Resource and Protection, vol. 3, no. 7, pp. 522-530, 2011.

[36] Environmental Protection Agency (EPA), Parameters of Water Quality: Interpretations and Standards, Environmental Protection Agency (EPA), Wexford, Ireland, 2001. 
[37] S. Loko, K. E. Ahoussi, B. Y. Koffi et al., "Microbiological and physico-chemical quality of groundwater from artisanal sites of mining exploitation in the South-West of Côte d'Ivoire: case of the area of Hiré," International Journal of Scientific \& Engineering Research, vol. 4, no. 9, pp. 567-574, 2013.

[38] G. Nogueira, C. V. Nakamura, M. C. B. Tognim, B. A. A. Filho, and B. P. D. Filho, "Microbiological quality of drinking water of urban and rural communities, Brazil," Revista de Saúde Pública, vol. 37, no. 2, pp. 232-236, 2003.

[39] A. Olalemi and V. O. Dauda, "Monitoring of selected groundwater sources for fecal contamination using bacterial and viral fecal pollution markers," International Journal of Public Health Research, vol. 6, no. 3, pp. 83-92, 2018. 